\title{
Study on Gear Chamfering Method based on Vision Measurement
}

\author{
Jun Sun \\ College of Civil Engineering and Architecture, \\ Guizhou University of Engineering Science,Bijie,Guizhou 551700,China
}

\begin{abstract}
This article summarize gear parameter measurement method and machine vision measurement method, proposed the two non-contact type measuring method based on machine vision and laser sensors, and design scheme for the measurement of gear chamfering profile parameters. The whole structure of the gear chamfering measurement system include hardware design and software design of measurement system. Article initially realized two laser displacement sensor measurement precision measurement of visual localization based, and designed bevel gear measuring path contour measuring methods, and the system also enables multiple cross-sectional contour measuring visual orientation and gear chamfering gears.
\end{abstract}

KEYWORDS: non-contact measurement; chamfering gear; machine vision; laser sensor; non-contact measurement;

\section{INTRODUCTION}

Bevel gear is one of the parts of cars, tractors, motorcycles and other essential gearbox, it is basic component that achieve shift-oriented, motion passed. The main structure to be measured on the chamfered gear teeth on the end, due to the relative positions of the sectional limit measured chamfer lies, there is not a good measurement method that can measure tooth tip chamfer profile. The presence of existing measurement systems is difficult to locate, poor reproducibility, time-consuming, human factors and other issues in the measurement of such gear chamfering ${ }^{[1]}$.

Laser ranging technology has been widely used in modern surveying various areas, such as parts of the surface roughness of online measurement ${ }^{[2]}$, fast track detection ${ }^{[3]}$, the oil film thickness measurement ${ }^{[4]}$, speaker shell modal parameter measurements. This paper developed a non-contact profile measuring gear chamfering system based on laser ranging to meet the needs of gear chamfering profile measurements. Measurement system using laser displacement sensor ranging technology, combined with mobile platform motion $\mathrm{X}$ and $\mathrm{Y}$ directions in the horizontal plane, to achieve a cross-section profile measurement anywhere.

\section{The overall design of the measuring system}

The overall structure of the measurement system is shown in Figure 1, mainly comprises a laser displacement sensor, X-Y precision moving platform, positioning assembly tooth profile, rotating stage, marble base, the control box, the microcomputer, the print output device. The technical solution is:

(1) Contour chamfer longitudinal data collection realized by laser displacement sensor, laser displacement sensor to the chamfered surface height information into electrical signals and transmitted via the data cable to the data processor, the data processor is connected to the computer through a serial bus and communications.

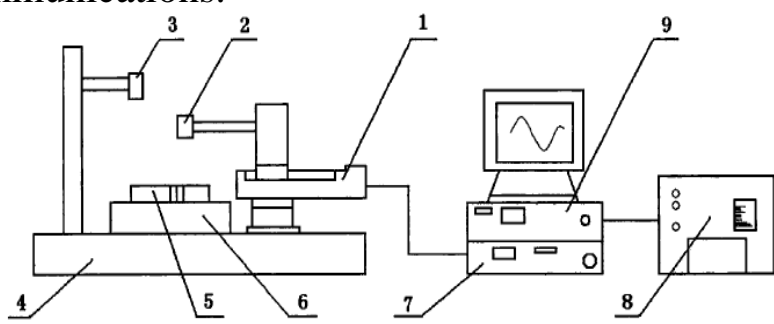

1. X-Y directions moving platform; 2.Laser sensor; 3.Vision sensor; 4. Marble platform;

5.Measured gear; 6.Working platform;

7.Control-box; 8.Printer; 9. Microcomputer

Fig.1 Structure of measurement system 
(2) X-Y-precision stepping motor driven mobile platform, taking incremental grating sensor as position detection aspects, and real time location and movement status is back to the control system, control system closed-loop control stepper motor in both directions according to the set procedures for the mobile platform. Computers to communicate via PCI bus mode and control systems.

(3) Use PC software platform to integrate various measurement modules measuring system, measuring software is modular design.

\section{Design of machine vision measurement module}

\subsection{The flow chart of machine vision measuring}

The main task of the machine vision measurement module is: image acquisition gear chamfering, image transfer to the computer, gear chamfering of the image is preprocessed through visual measurement software. After treatment, the center position information acquiring gear chamfering. The measurement flow chart of the machine vision measurement module is shown in Figure 2.
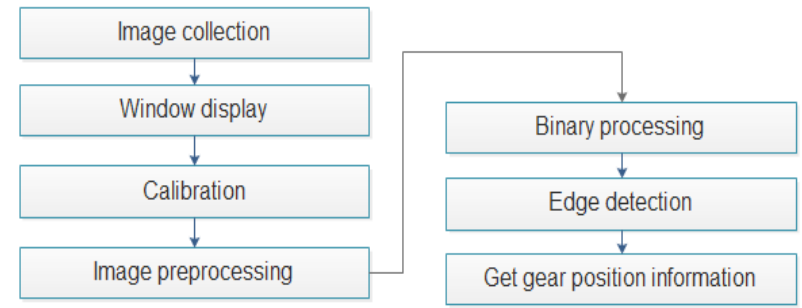

Fig. 2 The flow chart of machine vision measuring

The system gets the position information of gear chamfering, and stored position information of the gear, and passed to the motion control module.

\subsection{Motion control module}

The motion control module mainly includes the motion control of the $\mathrm{X}-\mathrm{Y}$ moving platform, the motion control and the interpolation motion control. The interpolation motion control can be realized to measure the tooth of any tooth. Therefore, there is no need to install the fixture in this measuring system.

There are two kinds of system measurement mode, the multi - Section Measurement and the standard survey. Multiple cross section measurement is designed according to the special requirements of gear chamfering detection mode of measurement and measurement standards applicable to general measurement task. In the measurement of gear chamfering process, multi section one-time measurement method, realizes the consistency and integrity measurement. The one time measurement strategy eliminates the interference of human factors, and reduces the random error of human operation, so as to achieve the purpose of accurate measurement. The point moving control is one of the basic functions of the mobile platform, and is used to realize the positioning of any position in the plane.

(1) Measurement travel calculation

The measurement length is the target stroke of the measurement system. The measurement length is input by the user, and the number of the pulses is converted to the following conversion.

$$
L=\frac{2 \pi n l}{\theta s}
$$

Type 1, L is the implementation of the components of the pulse number and $\mathrm{N}$ is a stepper motor subdivision, 1 is the distance measurement, unit: $\mathrm{mm}, \theta$ is fixed step distance of the motor, $\mathrm{s}$ is silk Du guide process.

(2) By the method of linear interpolation, the moving platform of $\mathrm{X}$ - Y direction is guided by the laser sensor along the moving track. Interpolation principle and features is:

Principle: $\mathrm{X}$ - Y direction mobile platform output shaft a feed pulse every time only along a coordinate, every move a step to deviation function calculation, judge the deviation between the deviation of the instantaneous 
coordinate movements in accordance with the provisions of, the mobile platform to reduce the deviation of the direction of feed, move on to the next point, the same judgment until all over. Make it close to the mobile trajectory. As shown in Figure 3:

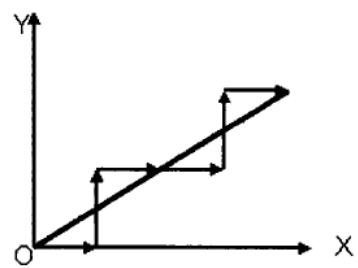

Fig.3 Linear interpolation

The calculation steps of the linear interpolation are as follows:

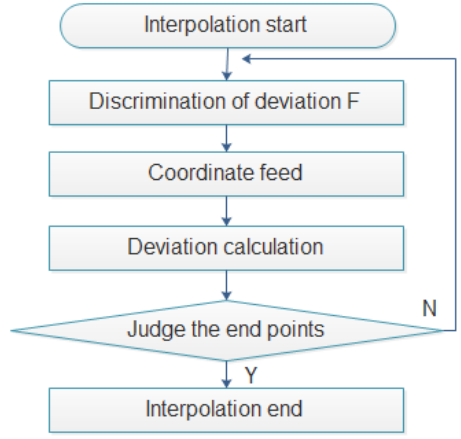

Fig.4 The flow chart of linear interpolation

(3) Measurement path design

The measurement path of the measurement system is shown in Figure 5. The measured tooth for any bevel gear, straight tooth was measured as the center line $0 \mathrm{P}, \mathrm{Pi}$ and $\mathrm{Pi}^{\prime}$ compared with the linear $0 \mathrm{P}$ symmetry. In Figure 5, dotted line represented motion path of bevel gear profile measurement, solid line represented measurement path in the process of measurement. The movement and measurement of laser displacement sensor along the path of $\mathrm{X}$-Y mobile platform.

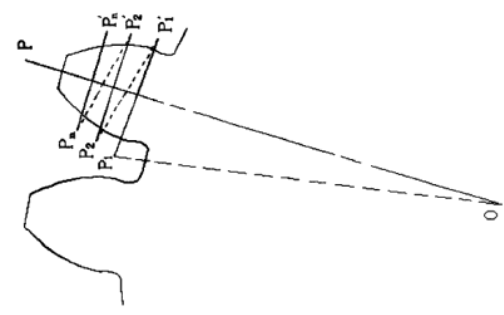

Fig.5 Measuring path

\subsection{Contour fitting}

Linear least square method is the most widely used parameter estimation method, which has important function in theoretical research and engineering application. The measurement software acquisition gear chamfering profile data, displayed in the evaluation area. To evaluate the gear chamfering profile parameters, the need for contour fitting. This system relates to the pointed arch angle measurement and round measuring and need high fitting a straight line and circle fitting.

The contour data obtained is a discrete point set, and the surface profile is $\mathrm{y}(\mathrm{x})$, and the target equation of the straight line is the least square:

$$
f(x)=y(x)=k x+b
$$

According to the least square principle, the square of the error is

$$
\varepsilon^{2}=\sum_{i=1}^{n}\left(y_{i}-f\left(x_{i}\right)\right)=\sum_{i=1}^{n}\left(y_{i}-k x_{i}-b\right)
$$

The sum of the error squares is the minimum, $\mathrm{K}$ and $\mathrm{b}$ respectively partial derivative and partial derivative is zero order.

$$
\frac{\partial \varepsilon^{2}}{\partial k}=\frac{\partial \varepsilon^{2}}{\partial b}=0
$$

Solving linear coefficient $\mathrm{k}$ to obtain objective and $\mathrm{b}$. The $\mathrm{k}$ and $\mathrm{b}$ can get into (1) a linear least squares fit equation.

\section{The experimental results and error analysis}

In order to test the reliability of vision measurement software in gear positioning machine vision, article use standard round (as shown in Figure 6) to carry out inspection experience. The standard round in the calibration plate, the use of cameras capture 10 images, each of these 10 image processing and measurement center. The results shown in Fig.7. 


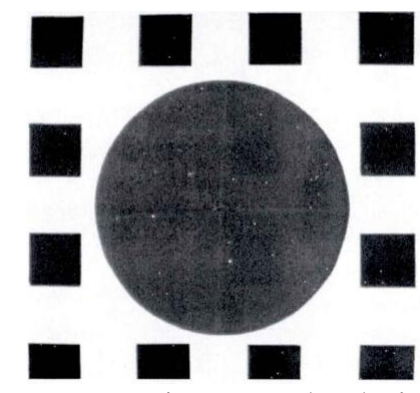

Fig.6 Measuring standard circle

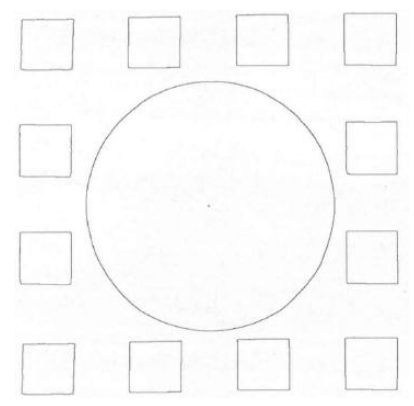

Fig.7 The result of measuring standard circle

Analysis of the experimental data results are shown in Table 1.

Table 1 the measuring results of standard circle center

\begin{tabular}{c|c|c|c|c|c}
\hline \multicolumn{2}{c|}{ X coordinates $(\mathrm{mm})$} & \multicolumn{3}{c}{ Y coordinates $(\mathrm{mm})$} \\
\hline Measurement & Theoretical valu & \multicolumn{1}{l|}{ Deviation } & Measurement & Theoretical & Deviation \\
\hline 74.0869 & 74.0000 & 0.0869 & 79.1384 & 79.0000 & 0.1384 \\
\hline 74.1563 & 74.0000 & 0.1563 & 79.1845 & 79.0000 & 0.1845 \\
\hline 74.0832 & 74.0000 & 0.0832 & 79.0587 & 79.0000 & 0.0587 \\
\hline 74.1409 & 74.0000 & 0.1409 & 79.0723 & 79.0000 & 0.0723 \\
\hline
\end{tabular}

The results showed that the experimental error be in $0.2 \mathrm{~mm}$, errors by system errors are mainly random errors. Such as effect by the light source of experimental systems, the camera distortion and other effects of the experiment. Whereby the end of the measurement shows that the larger the light source and camera distortion effect on the feasibility of the experimental system of measurement.

The paper can be chamfered gear chamfering gears based on machine vision guided laser measuring system. Cut and measure, more cut and measure and cut an arc measured. More cut and measure $\mathbf{M}$ and arc cross section measurements shown in Figure 8.
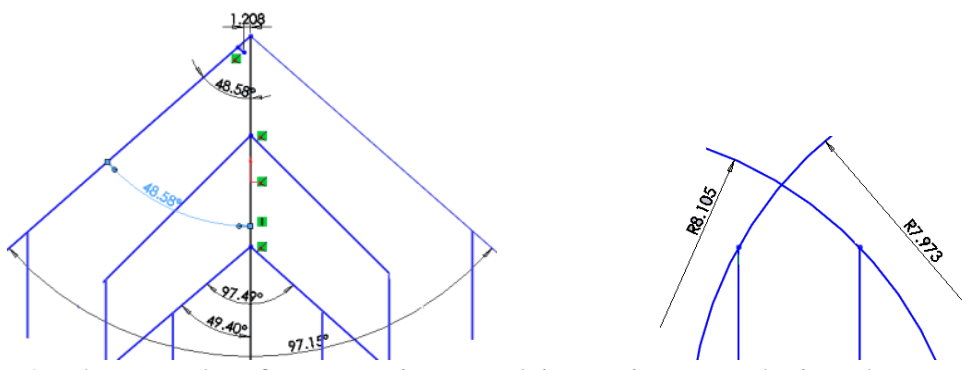

Fig. 8 The result of measuring multi-sections and circular-section

Standard gauge block (plug) is continuous measurements $\mathrm{H}$ times, each - times the actual measurement value, the standard values, error results are shown in Table 2.

Table 2 The accuracy verification of measurement system

\begin{tabular}{l|c|c|c}
\hline \multicolumn{2}{c|}{ Standard quantity block $(4 \mathrm{~mm})$} & Gauge $(18 \mathrm{~mm})$ & \\
\hline Actual measurement $(\mathrm{mm})$ & Error $(\mathrm{mm})$ & Actual measurement $(\mathrm{mm})$ & Error $(\mathrm{mm})$ \\
\hline 3.9920 & 0.0080 & 18.0270 & 0.0270 \\
\hline 3.9960 & 0.0040 & 18.0360 & 0.0360 \\
\hline 3.9910 & 0.0090 & 18.0250 & 0.0250 \\
\hline 3.9890 & 0.0110 & 18.0240 & 0.0240 \\
\hline
\end{tabular}


Standard gauge block measurement results show that the object width measurement system errors are less than $0.011 \mathrm{~mm}$; plug gauge measurement results show that the error measurement system are within the cylinder $0.036 \mathrm{~mm}$.

\section{Conclusions}

The paper use machine vision measurement method and two-stage non-contact measurement method based on laser sensor. Non-contact measurement methods have become an important means of precision measurement, gradually will replace the contact measurement methods. Non-contact measuring method have a high measurement speed, visualization, measurement, without damage etc. The two-stage method proposed in the paper includes non-contact measurement collection of machine vision positioning measurement and precision measurement laser displacement sensor, to achieve a fast, high-precision measurement method.

\section{Acknowledgement}

Bijie university Joint Fund Project(Guizhou science joint J LKB[2012]08)

\section{Reference}

[1] Li, Wang Li, high Xiaorong, et al. Hand-rail shape geometry measuring system [J] Optical Engineering, 2005,12 (32): 94-96.

[2] Hao Jianhua, Zhao Jianlin, et al. Using laser triangulation method to improve the detection accuracy of three-dimensional surface model method [J]. Optical technology, 1998,3 (2): 37-39.

[3] Liu Yong, Huang Jie, et al. Non-contact geometry of large forgings Online Measurement System [J]. China Measurement Technology, 2008,34 (5): 116-118.

[4] Wang Ying. Typical ball screw drive system failure analysis [J]. Precise Manufacturing \& Automation, 2008, 4, 29-34. 